\title{
ON UNSMOOTHABLE DIFFEOMORPHISMS
}

\author{
BY JENNY HARRISON \\ Communicated April 1, 1975
}

There exists on any two-manifold $M$ a $C^{r}$ diffeomorphism $f$ which is not topologically conjugate to any $C^{r+1}$ diffeomorphism for every $r \geqslant 0$.

The basic construction is on the annulus and consists of a sequence of annuli the sides of which are fixed and the center circles of which are rotated. The annuli converge to a fixed circle. After carefully specifying the rotation number of each center circle the widths of the annuli can be chosen so as to make the function $C^{r}$ but they cannot be wide enough for $f$ to be $C^{r+1}$ at the limit circle. Since the speed of the rotation cannot be effectively altered under a topological conjugacy there is no way to make the function $C^{r+1}$.

By suspending we obtain examples of foliations on $M \times S^{1}$ which are $C^{r}$ but cannot be made $C^{r+1}$.

The full details of the construction and the proof of invariance under a topological conjugacy are given in [1].

I am informed that C. Fefferman and W. Thurston have also constructed such an example (unpublished).

\section{REFERENCE}

1. Jenny Harrison, Unsmoothable diffeomorphisms, Warwick University Notes (to appear).

MATHEMATICS INSTITUTE, UNIVERSITY OF WARWICK, COVENTRY CV4 7AL, ENGLAND 